\title{
The issue of exposure to residual radiation of A-bomb survivors with estimates of very low primary radiation dose
}

\author{
Yoshisada Shibata
}

Received: 15 October 2008/ Accepted: 2 December 2008/Published online: 29 January 2009

(C) The Japanese Society for Hygiene 2009

The challenging publication by Masaru Miyao and colleagues in the latest issue of Environmental Health Preventive Medicine [1] attracted the attention of the mass media, and the results were reported sensationally by some newspapers; the headlines of The Mainichi (4 August 2008) and The Yomiuri (5 August 2008), respectively, read "A high cancer mortality even in A-bomb survivors exposed to low-dose radiation Hiroshima A-bomb. Up to 2.7 times higher than the non-exposed. Survey by Nagoya University and others" and "Hiroshima A-bomb. A high cancer mortality even in A-bomb survivors exposed to extremely low dose radiation. Survey by Nagoya University and others. Death from leukemia in male was 3 times higher than the non-exposed". Regrettably, however, the Watanabe et al. article [1] includes several defects, which calls into question their results and, hence, their conclusions.

The above-mentioned defects are as follows:

(1) Use of standardized mortality ratio (SMR) for comparisons

Note that the SMR varies depending on the age distribution of the target population even if the age-specific mortality rates are fixed for target population and standard population [2, 3]; the effects of the age distribution in the target population on the power of the hypothesis test are very large [3]. Furthermore, a comparison of SMRs among different populations may sometimes be invalid [2, 3]. This is because each SMR is standardized to the age distribution of its own population, which may differ between

\section{Y. Shibata $(\square)$}

Atomic Bomb Disease Institute, Graduate School

of Biomedical Sciences, Nagasaki University,

1-12-4 Sakamoto, Nagasaki 852-8523, Japan

e-mail: yshibata@nagasaki-u.ac.jp populations. The difference in the age structure of the populations being compared can create unseen, but real, biases. The authors should have compared the mortality directly between the target population and a common standard population, although the adequacy of the standard population should be discussed. Also note that a relatively large portion of the life span study (LSS) subjects have been living in areas other than Hiroshima or Okayama prefecture. The statement in the sixth paragraph of the Discussion does not indicate the comparability of LSS-H group with the two standard populations; it only indicates the comparability of the two standard populations.

(2) Confusion of the mortality study with the incidence study of the LSS

The exposed LSS subjects were selected in statistically appropriate ways from 284,000 people who responded "Yes" to the question "Were you in Hiroshima or Nagasaki at the time of the atomic bombing?" administered at the National Census of 1950. The non-exposed subjects were randomly selected using the rice roster and others from those who were not in Hiroshima or Nagasaki at the time of the atomic bombing but began to live in the cities afterwards. The death certificates of all subjects have been collected unless they have lost Japanese citizenship. Thus migration of LSS subjects, even from Japan, does not affect the quality of mortality data unless they lose Japanese citizenship. Therefore, the following statement in the eighth paragraph of the Discussion does not apply in this study: "However, the LSS-H group was estimated to have migrated somewhat, although the effect of migration was adjusted using the LSS cancer incidence data." It actually is the opposite; the cancer incidence data require adjustment for out-migration from Hiroshima or Nagasaki prefecture. Furthermore, note that the quality of mortality 
data is essentially the same as that of the vital statistics they used, and therefore the statement in the second paragraph of the Discussion is not correct either, and the statement in the third paragraph of the Discussion also applies to the cancer deaths in their standard populations.

\section{(3) Misunderstanding the results of the LSS study}

The major objective of the LSS study is estimation of radiation risks. When the construction of the LSS cohort was launched, the statistical techniques available were rather primitive compared with current techniques, and the cohort was designed for appropriate inter-comparison of the exposed group with the non-exposed group and intracomparisons in the exposed group. The NIC (not in city) group is the non-exposed group, which the authors may call the genuine control group. However, the NIC group was found by an LSS mail survey to be significantly different from the exposed group with regard to socio-economic status and lifestyle, which affect both cancer and noncancer risks [4]. Combined with the availability of more sophisticated statistical analysis, e.g. Poisson regression analysis, the main analysis of the LSS mortality and incidence studies are confined to the exposed group. Follow-up of the NIC group, however, has also been conducted. The LSS study usually presents the risk of radiation as the excess relative risk per $\mathrm{Sv}(\mathrm{ERR} / \mathrm{Sv})$ based on the cancer mortality or incidence observed in subjects who received from $<0.005 \mathrm{~Sv}$ to $4 \mathrm{~Sv}$, and it is not unusual scientifically to extrapolate the results to $0 \mathrm{~Sv}$. The SMR was not used as an analytical tool in the LSS study because of its inadequacy for risk estimation, and, hence, the statement in the eleventh paragraph of the Discussion does not apply.

\section{(4) Post hoc argument}

The authors concluded, on the basis of a relatively high SMR calculated using Hiroshima or Okayama prefecture residents for most cancer mortality in LSS-H group who received the primary radiation dose of less than $0.005 \mathrm{~Sv}$, that the results indicate a contribution of residual radiation that was not included in the exposure evaluation (last paragraph of the Discussion). However, the conclusion is based on a post hoc argument which was denied by Aristotle more than 2,300 years ago. The cause (radiation exposure in this case) should precede the results (death from cancer in this case), but the observed results do not necessarily indicate that they were due to that cause, unless the cause is unique. Furthermore, note that residual radiation exposure is ignored for all LSS subjects and it is difficult to imagine that the residual exposure should be higher in areas distant from the hypocenter than in those proximal to the hypocenter except the so-called "hot spot" areas.

The effects of the residual radiation should be investigated per se based on the most up-to-date knowledge of nuclear physics and whatever measurements are available. It is true that no significant dose of residual radiation has been proved up to the present in Hiroshima except in the Koi and Takasu areas, which are located about $3 \mathrm{~km}$ west of the hypocenter.

\section{References}

1. Watanabe T, Miyao M, Honda R, Yamada Y. Hiroshima survivors exposed to very low doses of A-bomb primary radiation showed a high risk for cancers. Environ Health Prev Med. 2008;13:264-70.

2. Rothman KJ. Modern epidemiology. Boston, Toronto: Little, Brown and Company; 1986.

3. Shibata Y. What does the standardized mortality ratio compare? Bulletin of the International Statistical Institute, Tome LIX, Book 2; 2001. pp. 137-8.

4. Kato H, Johnson KG, Yano K. Mail survey on cardiovascular disease study, Hiroshima and Nagasaki. ABCC TR 19-66; 1966. 